\title{
Dynamic Mechanical Analysis and High Strain-Rate Energy Absorption Characteristics of Vertically Aligned Carbon Nanotube Reinforced Woven Fiber-Glass Composites
}

\author{
Kiyun Kim, ${ }^{1}$ P. Raju Mantena, ${ }^{1}$ Seyed Soheil Daryadel, ${ }^{1}$ Veera M. Boddu, ${ }^{2}$ \\ Matthew W. Brenner, ${ }^{2}$ and Jignesh S. Patel ${ }^{2}$ \\ ${ }^{1}$ Composite Structures and Nano-Engineering Research, Department of Mechanical Engineering, University of Mississippi, \\ University, MS 38677, USA \\ ${ }^{2}$ U.S. Army Engineer Research and Development Center-Construction Engineering Research Laboratory (ERDC-CERL), \\ Champaign, IL 61821, USA
}

Correspondence should be addressed to Kiyun Kim; kky2729@gmail.com

Received 19 May 2015; Revised 16 July 2015; Accepted 21 July 2015

Academic Editor: Mircea Chipara

Copyright (c) 2015 Kiyun Kim et al. This is an open access article distributed under the Creative Commons Attribution License, which permits unrestricted use, distribution, and reproduction in any medium, provided the original work is properly cited.

\begin{abstract}
The dynamic mechanical behavior and energy absorption characteristics of nano-enhanced functionally graded composites, consisting of 3 layers of vertically aligned carbon nanotube (VACNT) forests grown on woven fiber-glass (FG) layer and embedded within 10 layers of woven FG, with polyester (PE) and polyurethane (PU) resin systems (FG/PE/VACNT and FG/PU/VACNT) are investigated and compared with the baseline materials, FG/PE and FG/PU (i.e., without VACNT). A Dynamic Mechanical Analyzer (DMA) was used for obtaining the mechanical properties. It was found that FG/PE/VACNT exhibited a significantly lower flexural stiffness at ambient temperature along with higher damping loss factor over the investigated temperature range compared to the baseline material FG/PE. For FG/PU/VACNT, a significant increase in flexural stiffness at ambient temperature along with a lower damping loss factor was observed with respect to the baseline material FG/PU. A Split Hopkinson Pressure Bar (SHPB) was used to evaluate the energy absorption and strength of specimens under high strain-rate compression loading. It was found that the specific energy absorption increased with VACNT layers embedded in both FG/PE and FG/PU. The compressive strength also increased with the addition of VACNT forest layers in FG/PU; however, it did not show an improvement for FG/PE.
\end{abstract}

\section{Introduction}

Development of novel, light-weight, high-strength, and hightemperature resistant materials has been the focus of increased research for many applications [1] such as aerospace and automobile structures, where the material experiences severe thermal gradients and requires high flexural rigidity and high vibration damping. Due to the increasing demand of required conflicting properties, the newly developed functionally graded materials (FGM) have been a broad research area on account of their tailored properties $[2,3]$. Investigation of carbon nanotubes' (CNT) role in CNT-polymer composites and their dynamic behavior can also help to develop such FGM [4]. CNT can enhance the mechanical properties of FGM, due to their unique material properties, such as high stiffness, strength, and toughness $[5,6]$. These nano-enhanced FGM are being considered for blast/ballistic protective structures and other armor applications.

Zeng et al. [7] studied the mechanical properties of VACNT based sandwich composites using DMA. It was observed that VACNT based sandwich composites showed higher flexural rigidity and damping compared to samples consisting of carbon fiber fabric stacks without VACNT. In previous research [8] conducted at the Blast and Impact Dynamics laboratory, University of Mississippi, the dynamic mechanical behavior and high strain-rate response of a FGM system consisting of VACNT grown on a silicon $(\mathrm{Si})$ wafer substrate have been investigated. It was found that 
$10+3$ layers of FG/CNT composite fabrication

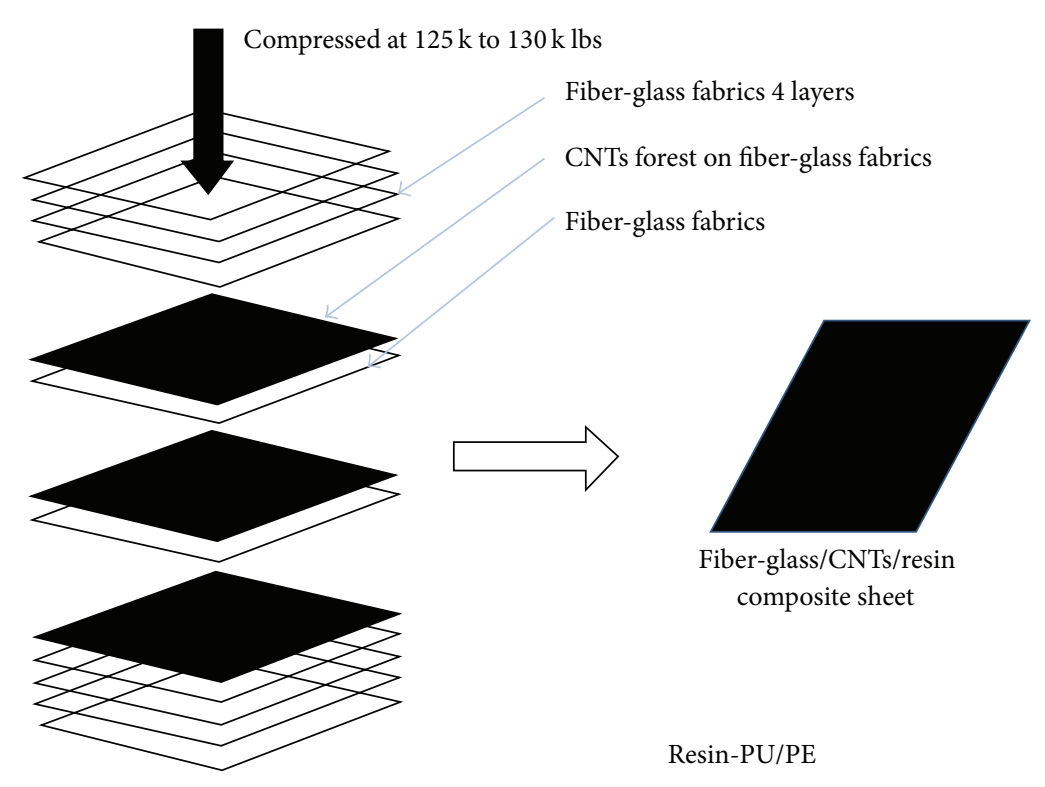

FIGURE 1: Schematic of the FG/PE/VACNT and FG/PU/VACNT samples with 10 layers of woven fiber-glass + 3 layers of embedded VACNT [9].

VACNT forests grown on Si wafer substrate exhibited significantly higher flexural stiffness, damping, and specific energy absorption.

In the current study, dynamic mechanical behavior and energy absorption characteristics of a VACNT-enhanced FGM system are investigated. VACNT forests were grown on the woven FG fabric layers with two different thermoset resin systems, PE and PU, and embedded within the specimens. Two different experimental techniques, DMA and SHPB, were used for evaluating their dynamic properties.

\section{Materials and Methods}

2.1. Specimen Preparation. 10-layer woven FG fabrics with $\mathrm{PE}$ resin (E15-8082) and 10-layer woven FG fabrics with PU resin (3475 Urethane Casting) were prepared as baseline composites (FG/PE and FG/PU) by the autoclave process.

Carbon nanotubes were grown on $12^{\prime \prime}$ by $12^{\prime \prime}$ sheet of desized glass fabric. Three sheets of the fabric were arranged on each of three racks in a steel chamber $\left(\sim 18^{\prime \prime} \times 18^{\prime \prime} \times 9^{\prime \prime}\right)$ which was heated to about $650^{\circ} \mathrm{C}$. A solution of $4 \%$ by wt. ferrocene in $\mathrm{m}$-xylene was prepared to implement the floating catalyst fabrication of CNTs and fed through three inlet manifolds, each of which was externally heated to about $350^{\circ} \mathrm{C}$ to vaporize the solution. Nitrogen and hydrogen, at a flow rate of 2000 and $300 \mathrm{cubic} \mathrm{cm} / \mathrm{min}$, respectively, carried the vapor into the reaction chamber containing the glass fabrics. The fabrics were processed for $60 \mathrm{~min}$. The temperature in the reaction chamber, however, fluctuated $\left( \pm 50^{\circ} \mathrm{C}\right)$ significantly around $600^{\circ} \mathrm{C}$ during the CNT growth process. A total ferrocene/catalyst solution of about $300-400 \mathrm{~mL}$ was used. FG/PE/VACNT and FG/PU/VACNT specimens having
3 layers of VACNT grown/embedded within 10 layers of woven FG along with two different resin systems (PE and $\mathrm{PU})$ were fabricated, by hand lay-up and compression. A schematic of FG/PE/VACNT and FG/PU/VACNT is shown in Figure 1. The composites were then cut into individual samples, as required for DMA $(60 \mathrm{~mm}$ long $\times 10 \mathrm{~mm}$ wide $\times$ $6 \mathrm{~mm}$ thick rectangular beams $)$ and SHPB $(6 \mathrm{~mm} \times 6 \mathrm{~mm} \times$ $6 \mathrm{~mm}$ square specimens) tests. More details of the specimen preparation and fabrication are given in [9].

2.2. DMA Technique. Dynamic mechanical behavior of FG/ PE, FG/PU, FG/PE/VACNT, and FG/PU/VACNT specimens was investigated using a TA Instruments Model Q800 DMA [10]. Rectangular beam specimens, with $60 \mathrm{~mm}$ in length, $10 \mathrm{~mm}$ in width, and $6 \mathrm{~mm}$ in thickness, provided by ERDC-CERL (U.S. Army Engineer Research and Development Center-Construction Engineering Research Laboratory (ERDC-CERL), Champaign, IL 61821, USA) were used for this study. Mechanical properties such as storage modulus (flexural stiffness), loss modulus (energy dissipation), $\tan \delta$ (inherent damping), and glass transition temperature $\left(T_{g}\right)$ were obtained in the three-point oscillatory bending mode. Roller pins on each side (with a span of $50 \mathrm{~mm}$ ) were used to support the specimens and force applied in the middle of the span. All the specimens were subjected to $1 \mathrm{~Hz}$ single frequency with $10 \mu \mathrm{m}$ midspan displacement amplitude. For these experiments, only the heating temperature ramp (from ambient $30^{\circ} \mathrm{C}$ to $200^{\circ} \mathrm{C}$ ) was implemented, with the temperature elevated in $2^{\circ} \mathrm{C} / \mathrm{min}$ steps up to the final temperature. Poisson's ratio of 0.3 is input as a constant parameter for all samples. 


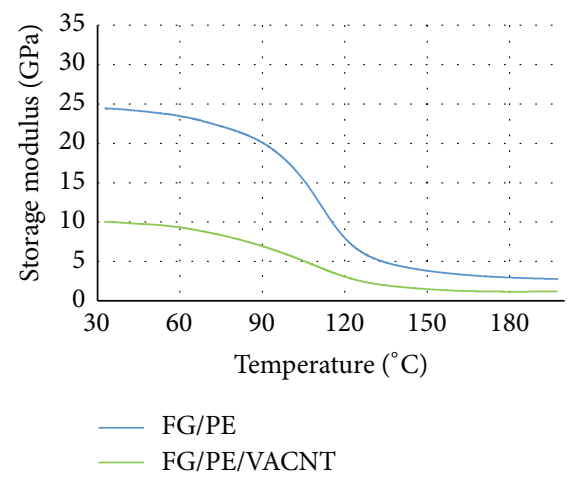

(a)

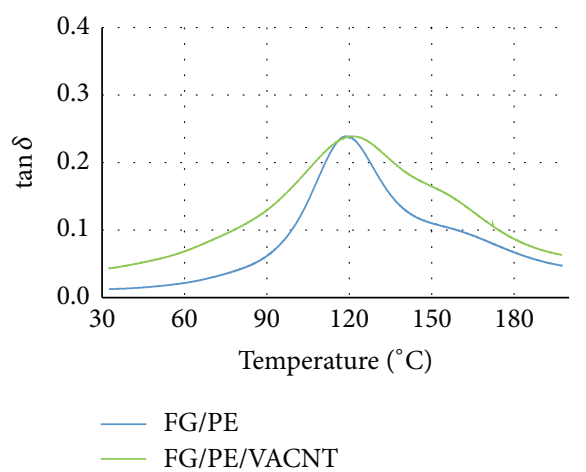

(c)

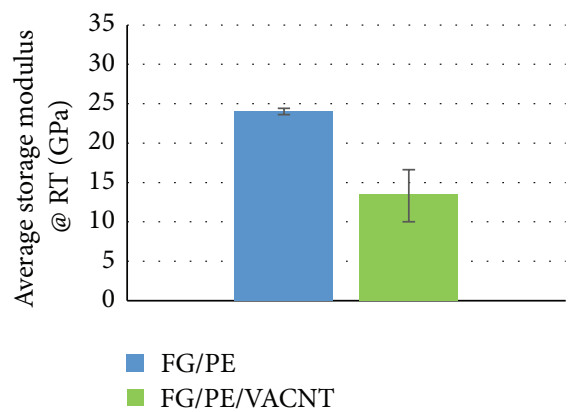

(b)

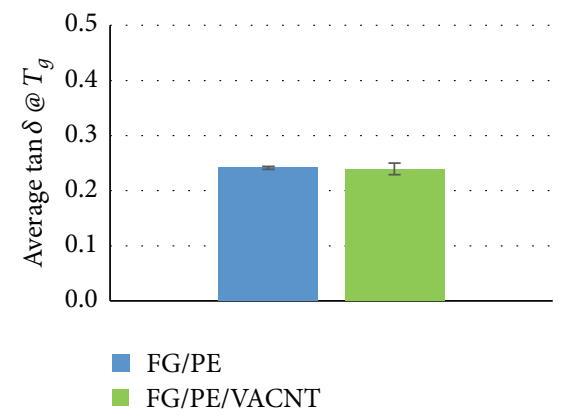

(d)

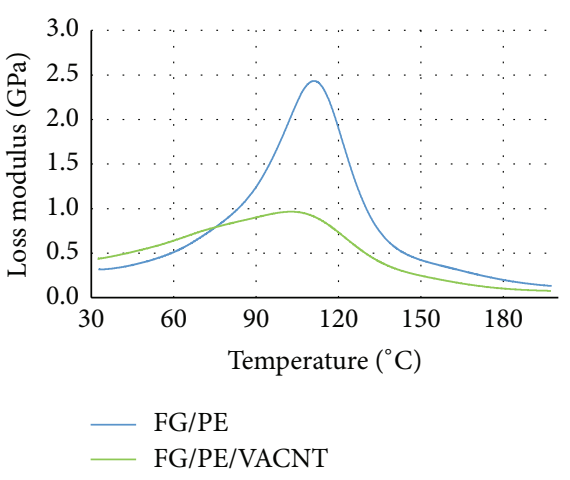

(e)

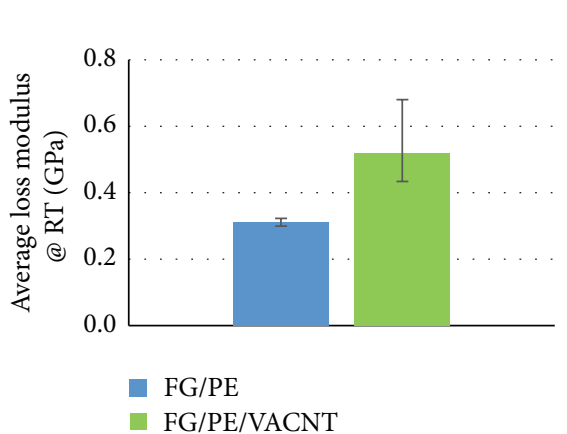

(f)

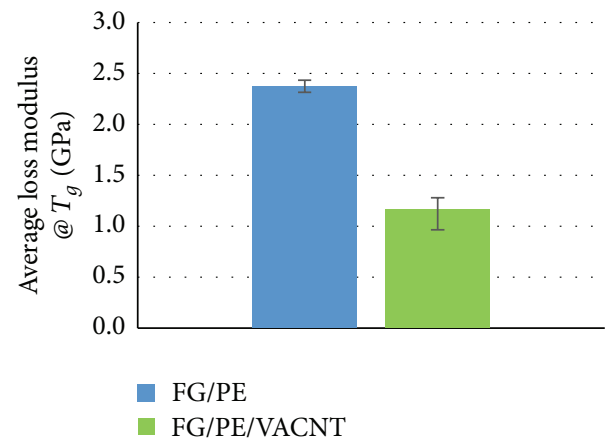

(g)

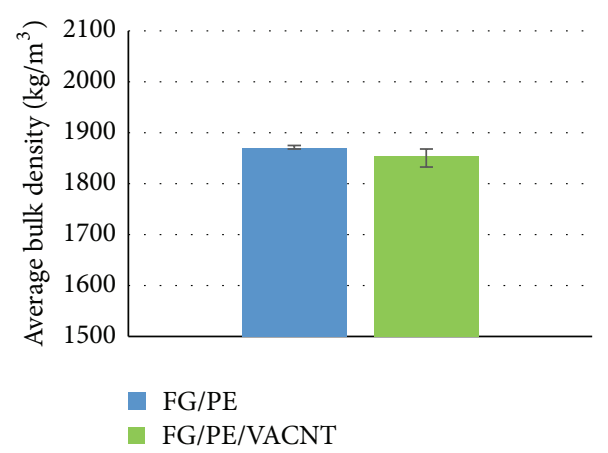

(h)

FIGURE 2: DMA response of 10-layer woven fiber-glass with PE resin (FG/PE) and 10-layer woven fiber-glass with PE resin along with embedded 3 layers of VACNT (FG/PE/VACNT): (a) storage modulus, (b) storage modulus at RT, (c) damping loss factor, (d) damping loss factor at $T_{g}$, (e) loss modulus, (f) loss modulus at RT, (g) loss modulus at $T_{g}$, and (h) average bulk density of specimens (three each). 


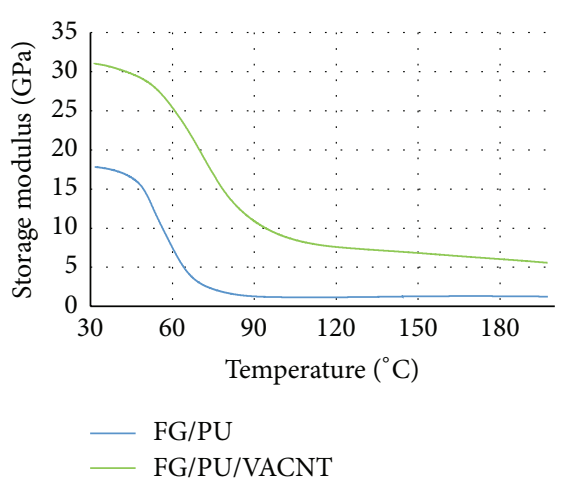

(a)

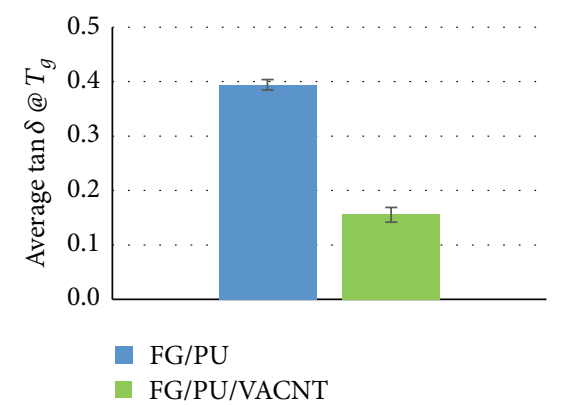

(d)

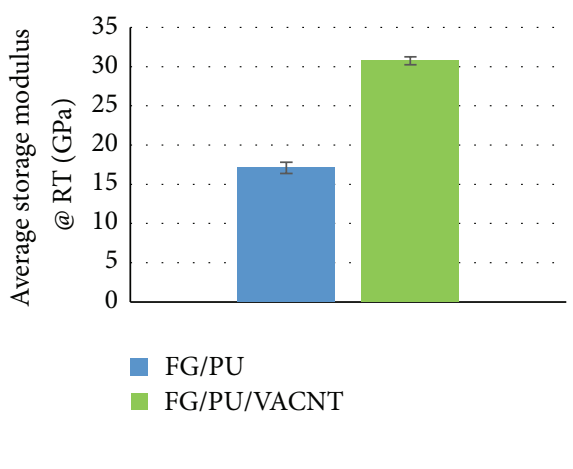

(b)

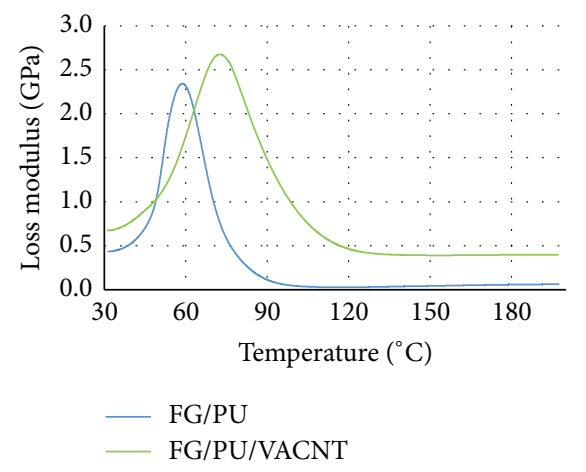

(e)

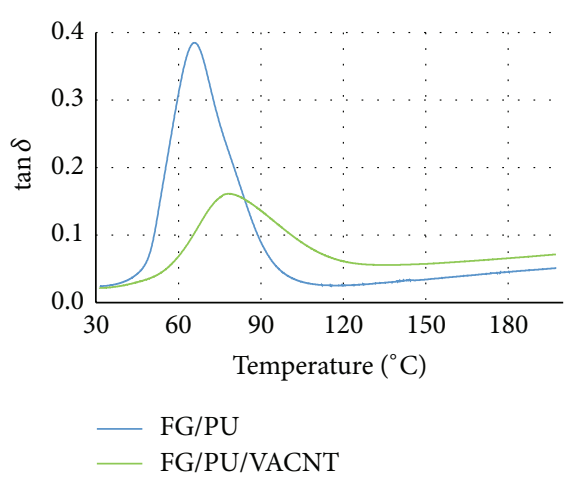

(c)

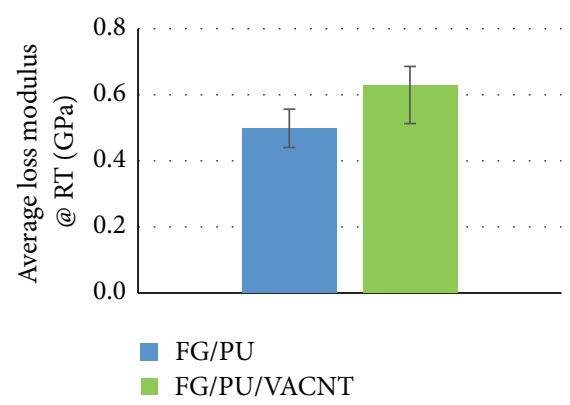

(f)

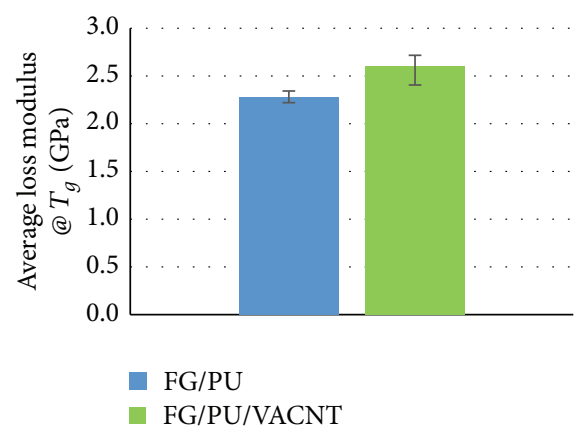

(g)

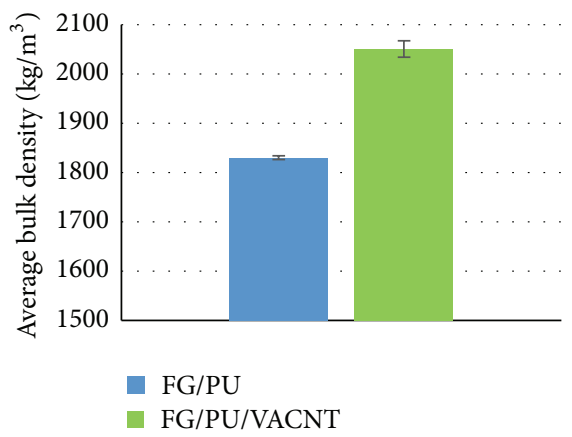

(h)

FIgURE 3: DMA response of 10-layer woven fiber-glass with PU resin (FG/PU) and 10-layer woven fiber-glass with PU resin along with embedded 3 layers of VACNT (FG/PU/VACNT): (a) storage modulus, (b) storage modulus at RT, (c) damping loss factor, (d) damping loss factor at $T_{g}$, (e) loss modulus, (f) loss modulus at RT, (g) loss modulus at $T_{g}$, and (h) average bulk density of specimens (three each).

2.3. SHPB Technique. The high strain-rate compressive tests were conducted on FG/PE, FG/PU, FG/PE/VACNT, and FG/PU/VACNT specimens using a modified SHPB in the Blast and Impact Dynamics Lab at the University of Mississippi, MS. Aluminum bars of $19.02 \mathrm{~mm}$ diameter were used as striker, incident, and transmission bars. Square specimens were cut precisely $(6 \mathrm{~mm} \times 6 \mathrm{~mm} \times 6 \mathrm{~mm})$ and tested with compression SHPB apparatus to evaluate the dynamic mechanical properties such as compressive strength, specific energy absorption, and rate of specific energy. Polyurea pulse shaper was used to minimize the wave dispersion and achieve the required stress equilibrium [11]. Glycerin was also used for holding specimens in-between incident and transmission bar ends to minimize the interfacial friction.

\section{Results and Discussion}

3.1. DMA Results. The DMA experiments were conducted on FG/PE, FG/PU, FG/PE/VACNT, and FG/PU/VACNT samples (three samples each) to compare and investigate effects of the embedded VACNT layers on specimens' dynamic mechanical behavior. The output data graphs, the storage modulus (flexural stiffness), the loss modulus (energy dissipation), and the damping loss factor $(\tan \delta$, ratio of dissipated 


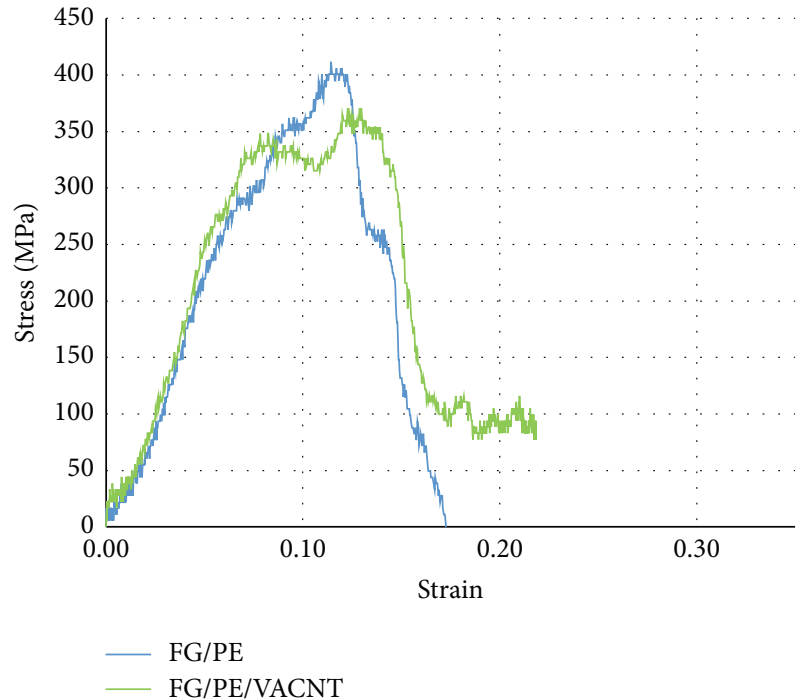

(a)

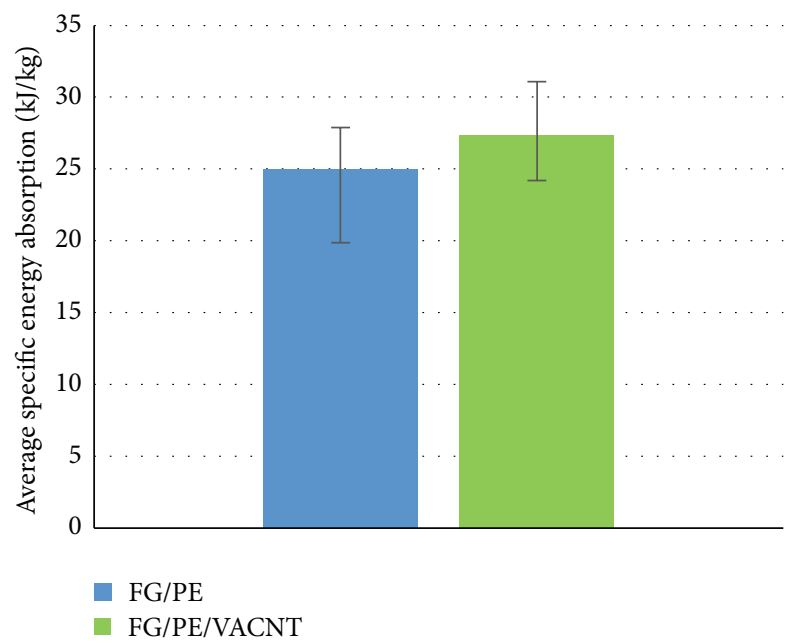

(c)

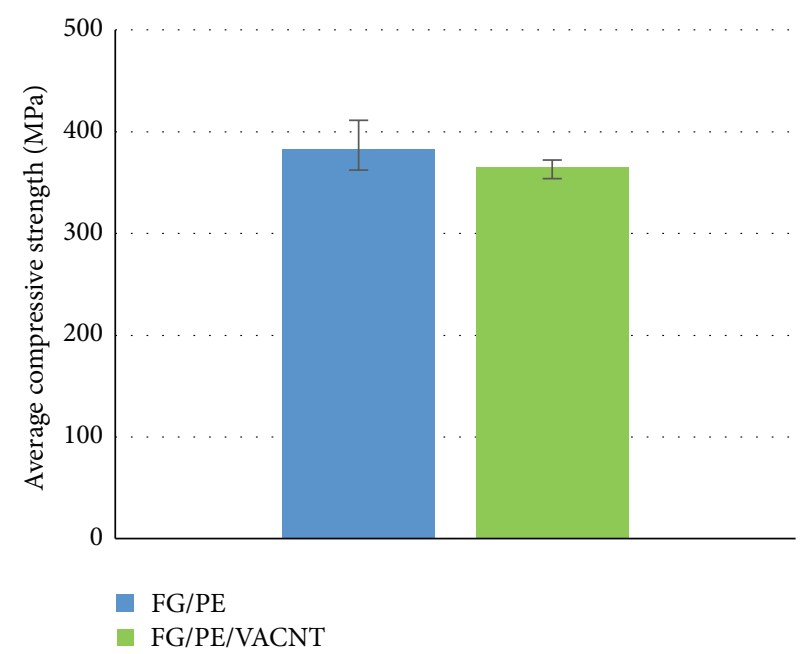

(b)

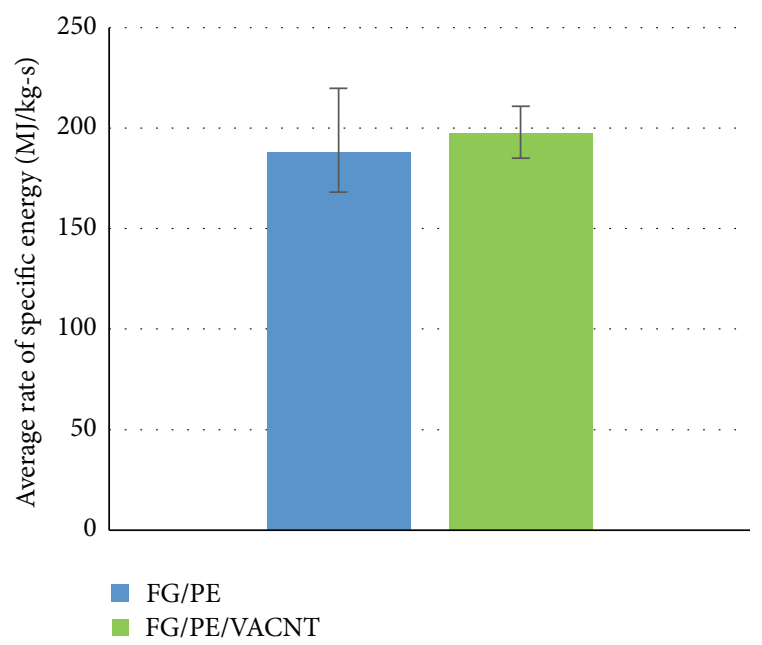

(d)

FIGURE 4: SHPB compression test response of FG/PE and FG/PE/VACNT composites evaluated over strain rate of 700 to 800/s: (a) stressstrain curve of FG/PE and FG/PE/VACNT, (b) compressive strength, (c) average specific energy absorption, and (d) rate of specific energy absorption.

energy to stored energy) for FG/PE and FG/PE/VACNT specimens are presented in Figure 2. As can be seen, at room temperature (RT) FG/PE/VACNT samples exhibited 40\% drop in storage modulus compared to the baseline FG/PE samples. The $\tan \delta$ of FG/PE/VACNT specimens over the test temperature range from $32^{\circ} \mathrm{C}$ to $200^{\circ} \mathrm{C}$ (or frequency, by the timetemperature correspondence principle for viscoelastic materials) appeared to be consistently higher (by about $60 \%$ ), but it was found to be similar as baseline FG/PE at $T_{g}\left(120^{\circ} \mathrm{C}\right)$. The loss modulus of FG/PE/VACNT was higher at RT compared to the baseline $\mathrm{FG} / \mathrm{PE}$, but it was much lower at $T_{g}\left(120^{\circ} \mathrm{C}\right)$. The average bulk density of FG/PE/VACNT was slightly lower than the FG/PE specimens. Glass transition temperature was almost the same for both composites $\left(120^{\circ} \mathrm{C}\right)$.

In Figure 3, the dynamic mechanical behavior of FG/PU/ VACNT and FG/PU specimens is compared. It was found that the storage modulus of FG/PU/VACNT increased (from about $18 \mathrm{GPa}$ to $31 \mathrm{GPa}$ at RT) in comparison with the baseline FG/PU samples. Additionally, FG/PU/VACNT exhibited higher loss modulus at both RT and $T_{g}$. The average bulk density and $T_{g}$ also increased. However, a significant drop in inherent damping $(\tan \delta)$ was observed at $T_{g}$ with the addition of VACNT layers (FG/PU/VACNT).

In contrast to previous investigation of VACNT grown on silicon wafer substrate [8], the addition of VACNT layers did not show an increase in damping loss factor $(\tan \delta)$ in $\mathrm{FG} /$ $\mathrm{PE} / \mathrm{VACNT}$ and FG/PU/VACNT samples. However, a significant drop in damping has been observed in FG/PU/VACNT samples. The hand lay-up with subsequent pressurization of the green samples may be the major factor in reduction of damping. Increased binding of the polymers with 


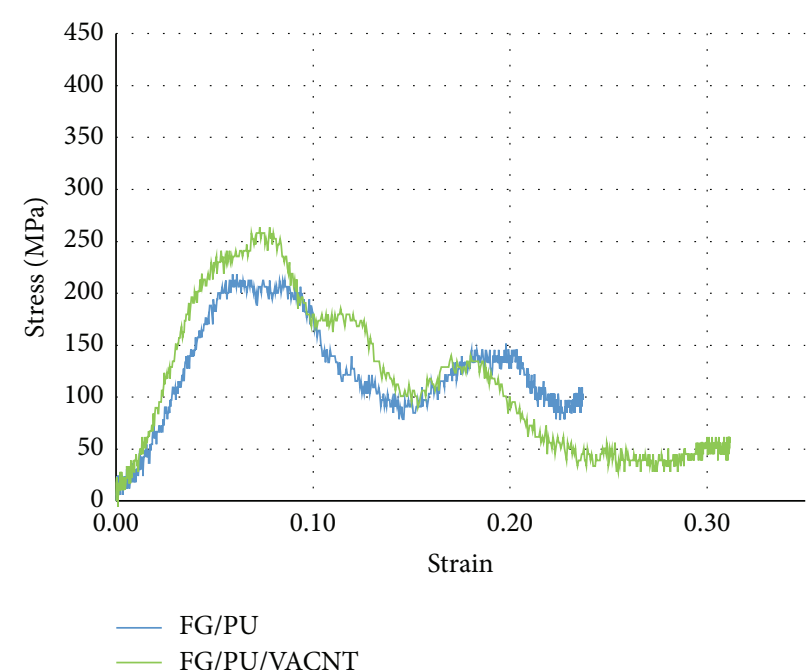

(a)

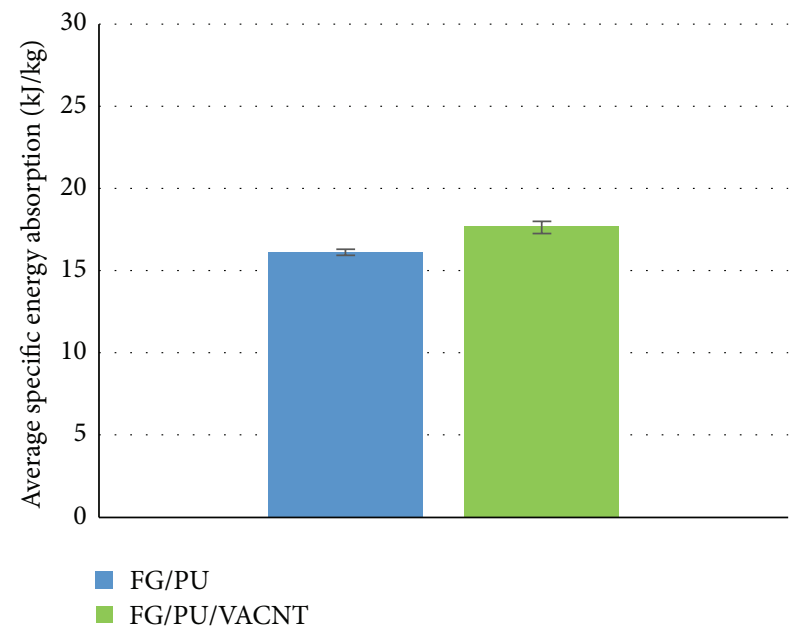

(c)

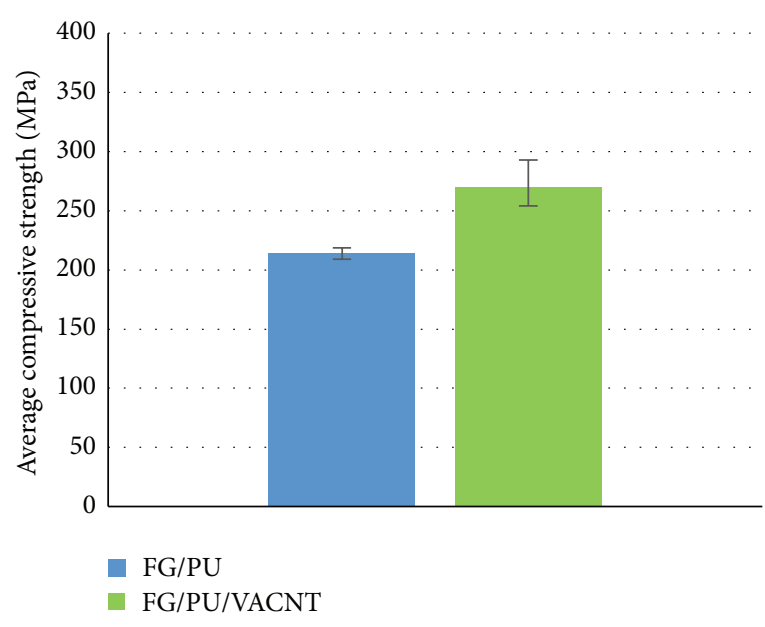

(b)

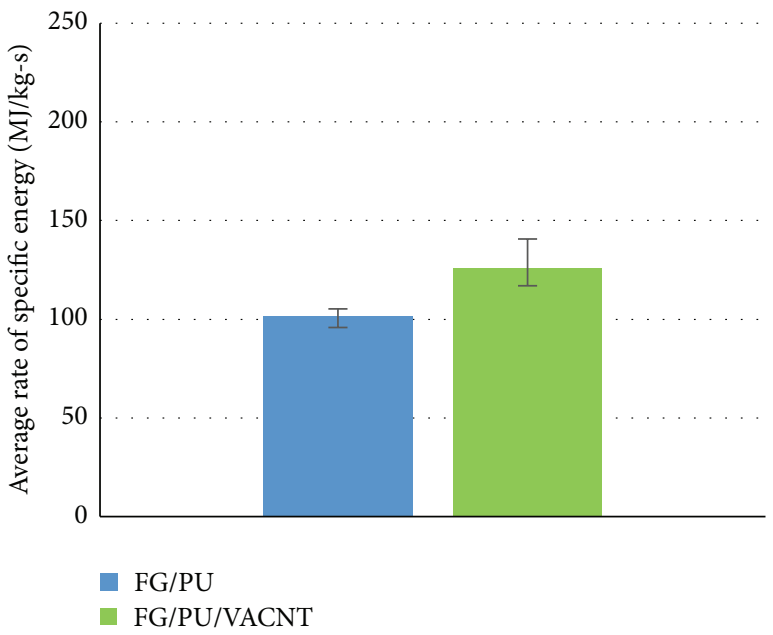

(d)

FIGURE 5: SHPB compression test response of FG/PU and FG/PU/VACNT composites evaluated over strain rate of 700 to 800/s: (a) stressstrain curve of FG/PU and FG/PU/VACNT, (b) compressive strength, (c) average specific energy absorption, and (d) rate of specific energy absorption.

the VACNT surface under pressure may also be contributing to the reduced damping.

3.2. SHPB Results. The compression SHPB apparatus was used to evaluate the energy absorption characteristics of FG/ PE, FG/PU, FG/PE/VACNT, and FG/PU/VACNT specimens. A typical SHPB compression response for FG/PE and FG/PE/ VACNT specimens at strain rate of 700 to $800 / \mathrm{s}$ is shown in Figure 4. FG/PE/VACNT displayed higher specific energy absorption and rate of specific energy compared to the baseline FG/PE. However, compressive strength in FG/PE/ VACNT was marginally lower than baseline FG/PE.

SHPB compression tests were also performed on baseline FG/PU and FG/PU/VACNT specimens at strain rate of 600 to $800 / \mathrm{s}$. Figure 5 shows typical stress-strain curves of FG/PU and FG/PU/VACNT and compares them in terms of average compressive strength, specific energy absorption, and rate of specific energy. FG/PU/VACNT exhibited higher specific energy absorption and rate of specific energy with respect to the baseline FG/PU. It was also found that the average compressive strength of FG/PU/VACNT increased about $30 \%$ with respect to FG/PU (from $214 \mathrm{MPa}$ to $270 \mathrm{MPa}$ ).

\section{Conclusions}

Dynamic mechanical behavior and energy absorption characteristics of 10-layer woven fiber-glass fabric with two different resin systems (PE and PU) have been investigated as the baseline; and effects of embedding VACNT layers within these baseline composites were studied. From DMA response, FG/PE/VACNT exhibited a significantly lower flexural stiffness at ambient temperature along with higher damping loss factor over the investigated temperature range with respect to baseline FG/PE. However damping loss 
factor was found to be similar as the baseline FG/PE at $T_{g}$. FG/PU/VACNT showed a significantly higher flexural stiffness at ambient temperature along with lower damping loss factor over the investigated temperature range compared to the baseline FG/PU. The loss modulus at RT increased with the addition of VACNT forest layers in both baseline composites (FG/PE and FG/PU), but only FG/PU/VACNT showed an increased loss modulus at $T_{g}$. From SHPB response, FG/PE/VACNT and FG/PU/VACNT showed improved specific energy absorption and rate of specific energy absorption compared to the baseline materials FG/PE and FG/PU. The compressive strength of FG/PU/VACNT increased by about $30 \%$ with the addition of VACNT forest layers.

\section{Conflict of Interests}

The authors declare that there is no conflict of interests regarding the publication of this paper.

\section{Acknowledgments}

The financial support from the U.S. Army Engineer Research and Development Center-Construction Engineering $\mathrm{Re}$ search Laboratory (ERDC-CERL), Champaign, IL, USA, Grant W9132T-12-P0057 is gratefully acknowledged. The authors would like to thank Dr. Brahma Pramanik for his help in performing the DMA and SHPB experiments.

\section{References}

[1] I. C. Finegan and R. F. Gibson, "Recent research on enhancement of damping in polymer composites," Composite Structures, vol. 44, no. 2-3, pp. 89-98, 1999.

[2] R. M. Mahamood, E. T. Akinlabi, M. Shukla, and S. Pityana, "Functionally graded material: an overview," in Proceedings of the World Congress on Engineering (WCE '12), vol. 3, pp. 15931597, London, UK, 2012.

[3] S. Thomopoulos, T. F. Martin, and R. N. Grishanin, Eds., Structural Interfaces and Attachments in Biology, Springer, New York, NY, USA, 2013.

[4] P. M. Ajayan and J. M. Tour, "Materials science: nanotube composites," Nature, vol. 447, no. 7148, pp. 1066-1068, 2007.

[5] J. N. Coleman, U. Khan, W. J. Blau, and Y. K. Gun'ko, "Small but strong: a review of the mechanical properties of carbon nanotube-polymer composites," Carbon, vol. 44, no. 9, pp. 1624$1652,2006$.

[6] E. T. Thostenson, Z. Ren, and T.-W. Chou, "Advances in the science and technology of carbon nanotubes and their composites: a review," Composites Science and Technology, vol. 61, no. 13, pp. 1899-1912, 2001.

[7] Y. Zeng, L. Ci, B. J. Carey, R. Vajtai, and P. M. Ajayan, "Design and reinforcement: vertically aligned carbon nanotube-based sandwich composites," ACS Nano, vol. 4, no. 11, pp. 6798-6804, 2010.

[8] P. R. Mantena, T. Tadepalli, B. Pramanik et al., "Energy dissipation and the high-strain rate dynamic response of vertically aligned carbon nanotube ensembles grown on silicon wafer substrate," Journal of Nanomaterials, vol. 2013, Article ID 259458, 7 pages, 2013.
[9] K. Kim, Dynamic mechanical analysis and high strain-rate energy absorption characteristics of vertically aligned carbon nanotube (VACNT) reinforced woven fiber-glass composites [M.S. thesis], The University of Mississippi, Oxford, Miss, USA, 2015.

[10] T. A. Instruments, "Q800 Dynamic Mechanical Analysis Brochure," 2010, http://www.tainstruments.com/pdf/brochure/ dma.pdf.

[11] W. W. Chen and B. Song, Split Hopkinson (Kolsky) Bar: Design, Testing and Applications, Springer, New York, NY, USA, 2011. 

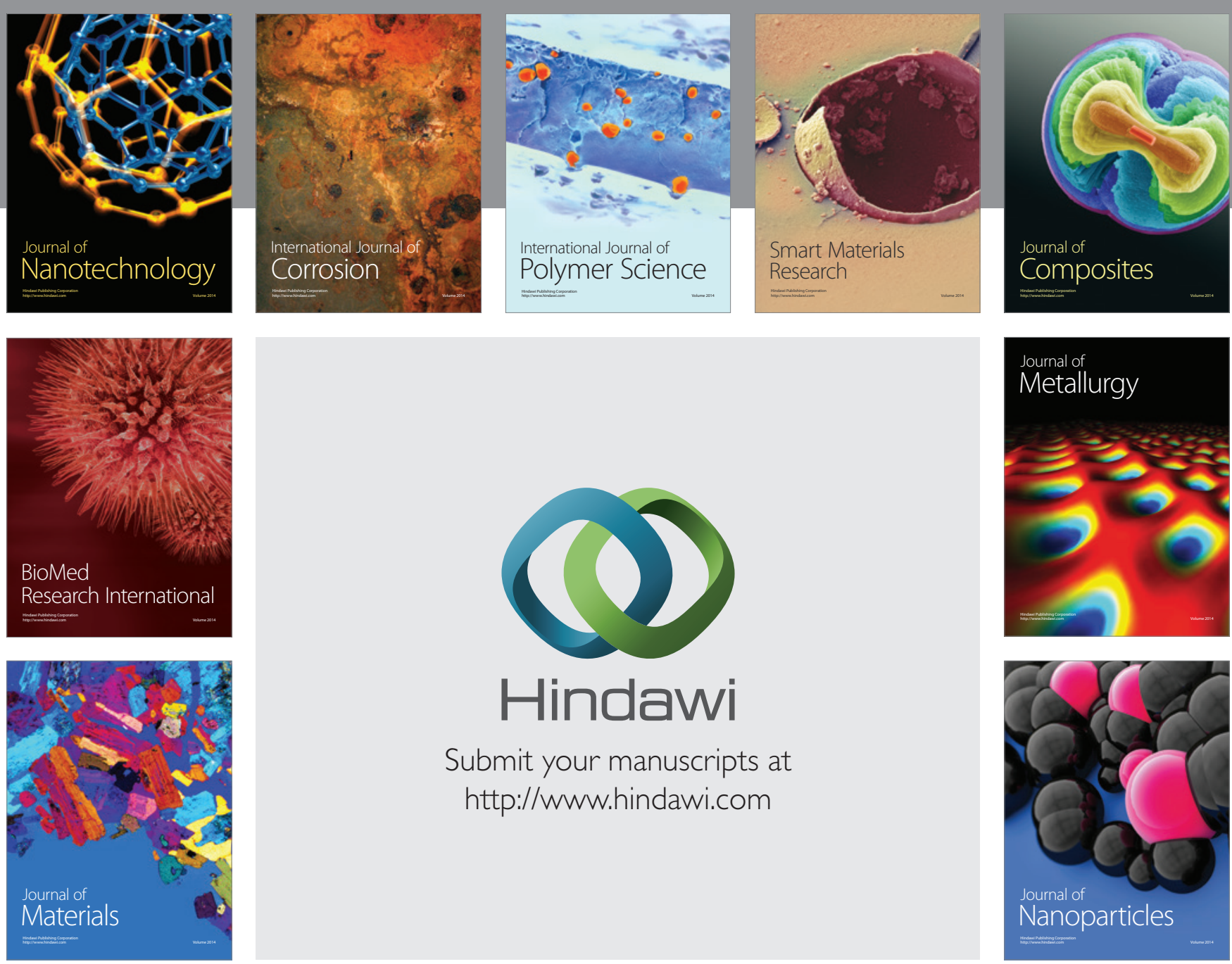

Submit your manuscripts at http://www.hindawi.com
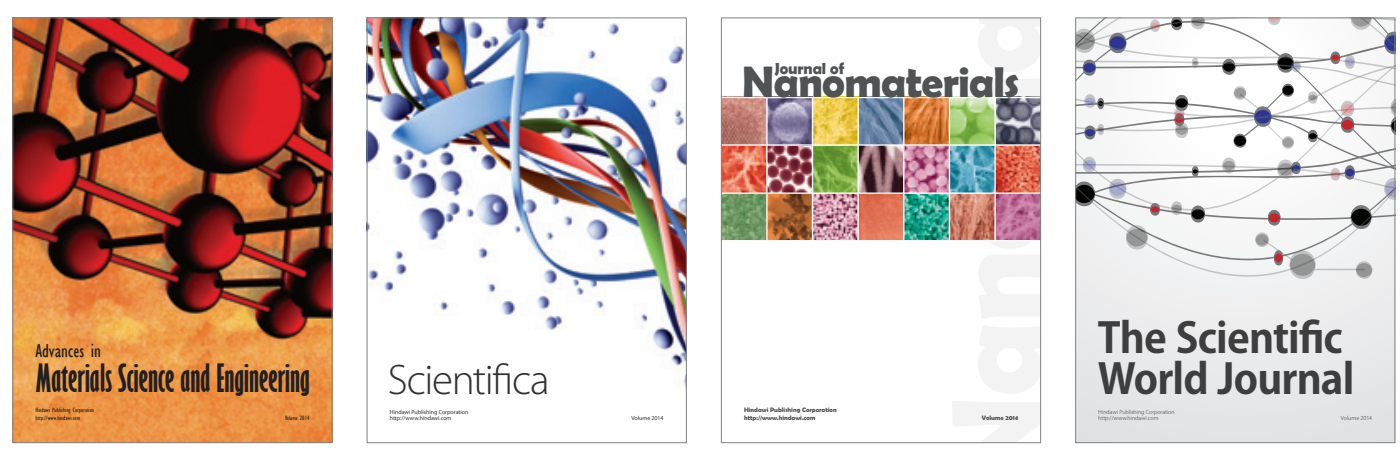

\section{The Scientific World Journal}
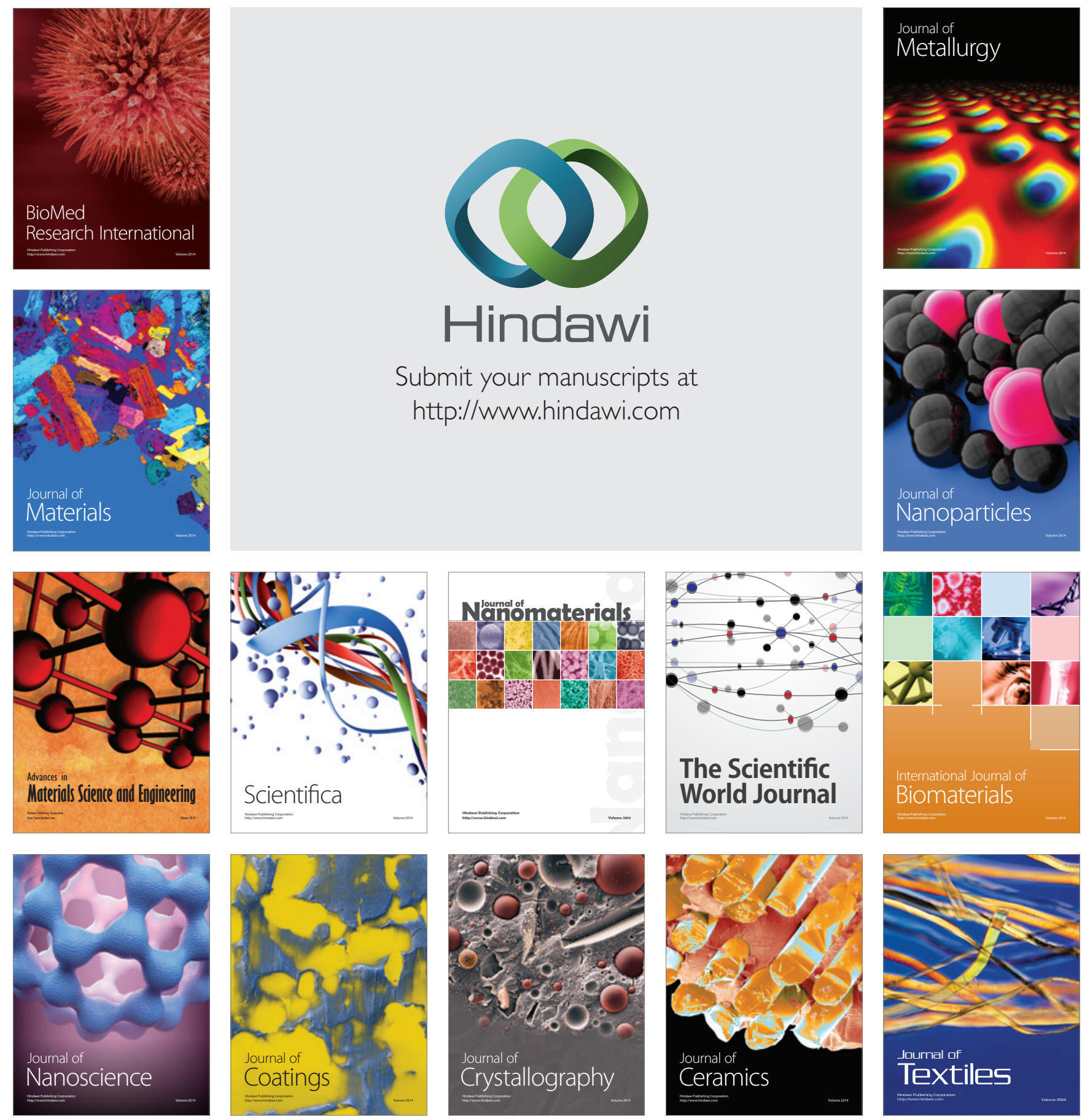